\title{
Utilising Computer Based Learning to Complement Class Teaching of Gross Anatomy
}

\author{
Rudi Klein $^{\mathrm{a}}$, Puspha Sinnayah ${ }^{\mathrm{a}, \mathrm{b}}$, Kate Kelly ${ }^{\mathrm{a}}$, Maxwell Winchester ${ }^{\mathrm{a}, \mathrm{c}, \mathrm{d}}$, Gayathri Rajaraman ${ }^{\mathrm{a}}$, \\ and Norman Eizenberg ${ }^{\mathrm{e}}$ \\ Corresponding author: Rudi Klein, rudi.klein@vu.edu.au \\ ${ }^{\mathrm{a}}$ First Year College, PO Box 14428, Victoria University, Melbourne VIC 8001, Australia \\ ${ }^{b}$ Institute for Health and Sport, Victoria University, Melbourne VIC 8001, Australia \\ 'Institute of Sustainable Industries and Liveable Cities, Victoria University, Melbourne VIC 8001, Australia \\ ${ }^{\mathrm{d} C}$ Copenhagen Business, Solbjerg Plads, Frederiksberg 2000, Denmark \\ ${ }^{\mathrm{e}}$ Melbourne Clinical School, University of Notre Dame, Werribee, Melbourne, VIC 3030, Australia
}

Keywords: Block model teaching, computer based gross anatomy learning, active learning

\begin{abstract}
This study examines the use of three computer programs designed for the study of gross anatomy; An@tomedia Online, AnatomyTV, and Thieme, as in-class learning support programs within the newly adopted "Block model" of teaching delivery at Victoria University. Victoria University is the first and only Australian university to have introduced a block model of education. With the introduction of 'The Block Teaching Model' the focus of the use of in-class time towards meaningful and active learning has become paramount. We used these programs as in-class student-centered inquiry based learning activity. The aim of this research was to investigate how the use of these programs impact on student engagement and student experience in their study of gross anatomy which is now offered in a time-compressed block teaching method. An opinion-based survey using the Qualtrics software was conducted at the end of each anatomy unit teaching block of semester 1 in 2019. The survey was distributed to all students enrolled in the unit. Results indicate that all programs used were viewed as making a positive contribution to student learning. The use of An@tomedia Online was particularly helpful in assisting first-year students studying gross anatomy with the interpretation of laboratory-based prosected cadaveric material within the new teaching context.
\end{abstract}

\section{Introduction}

Although the study of anatomy is seen as a cornerstone of a medical education (Estai \& Bunt, 2016), there have been concerns raised about the teaching resources and strategies used in teaching gross anatomy (Choi, Tamblyn, \& Stringer, 2008; Estai \& Bunt, 2016).

There are no shortage of options available for educators looking for electronic resources that can aid student learning in the anatomy field. Even a decade ago, there were over 100 websites, along with a handful of other online programs that could be used (Choi et al., 2008). While there is a large variety of excellent electronic resources currently available in providing useful supplementary material, only a few offer a more comprehensive, anatomically correct and relevant framework for study that could usefully complement the teaching of gross anatomy (Choi et al., 2008). This study adds to the body of knowledge of previous studies into the use of computer assisted learning (CAL) (Biasutto, Ignacio C, \& Esteban Criado del Río, 2006; Khot, Quinlan, Norman, \& Wainman, 2013; McNulty, Halama, \& Espiritu, 2004; McNulty, Sonntag, \& Sinacore, 2009; Reeves, Aschenbrenner, Wordinger, Roque, \& Sheedlo, 2004; Tam et al., 2010). 
This study examines the use of 3 different computer programs: An@tomedia Online (Eizenberg, Briggs, Barker, \& Grkovic, 2019), AnatomyTV (Primal-Pictures, 2019) and Thieme (Gilroy et al., 2019) (available on the University Library data base) specifically designed for the study of gross anatomy at undergraduate level. We chose these particular programs because of their immediate availability from the University library database, and because they are among the most widely used programs at tertiary institutions for this purpose. These readily available electronic resources now offer new opportunities to improve gross anatomy learning, when applied as a student-centred learning activity (Freeman et al., 2014; Michael, 2006) within a new method of teaching delivery called the block model recently adopted by Victoria University (McCluskey, Weldon, \& Smallridge, 2019).

In 2018, Victoria University moved all first-year units ("unit of study" is an Australian term which describes what is commonly called a "course" in North America, or a "module" in The UK or subject in NZ) from a regular 12 week semester, to a four week intensive block (McCluskey et al., 2019). Anecdotal evidence suggests the implementation of this intensive block model has been very successful (McCluskey et al., 2019), with an increase in student grades (Bolton, 2018; Cook, 2018) and a reduction in fail rates (Cook, 2018; Matchett, 2018) and higher student retention rates (Cook, 2018). A more mixed review of the benefits and limitations of such a model have been reported by others (Burton \& Nesbit, 2002; Clark \& Clark, 2000; Davies, 2006; Dixon \& O’Gorman, 2019).

While the delivery of intensive short courses at higher education is not new, there is little research validating its effectiveness or otherwise. While there is a reasonable volume of literature dealing with short or intensive courses per se there is little known about its impact at tertiary level. Time-compressed delivery of units certainly have their advantages for both Universities as well as students, hence their frequent use, but their growing application has been mainly limited to management schools (Davies, 2006). Very few or a complete lack of their routine application is found elsewhere in the Australian tertiary sector with the exception of the US, where the educational sector is arguably different. Existing research into this area of pedagogy has focused mainly on student perceptions towards a single intensive unit (Grant David, 2001; Kasworm, 2001) from which data was collected immediately following the completion of a unit, and thus may not reflect any long term trends of such teaching method (Daniel, 2000). In previous research, the assessment of a single unit at a single point in time also fails to capture any information on student preferences and learning styles. In-spite of this lack of empirical data comparing alternative delivery formats, intensive teaching formats have been reported to be more popular with students for certain units (Grant David, 2001; Scott, 2003). One noted aspect within our block model are the consistently high attendance rates for most units. This means that students should ideally be provided with a learning environment or activity that capture the benefits and dynamics existing within such environment. To this end, we attempted to harness the time allocated to each workshop to capture greater student engagement and student-centered learning and to develop a culture of community learning through group discussion so important in staying connected.

In this study, we used student-centered activities that contain active learning principles previously identified as being of fundamental importance to making the students learning experience enriching, rewarding, meaningful and worthwhile (Deslauriers, Schelew, \& Wieman, 2011; Freeman et al., 2014; Kift, 2009; Michael, 2006; Rathner, Hughes, \& Schuijers, 2013). The computer assisted learning programs were embedded within a blended learning design in which other resources were also available online. The parameters of this study are unique, being within the context of the block-teaching model, coupled with high attendance 
rates. For these reasons, we were interested in studying the combination of these two parameters as a platform for the effectiveness of student-centred resources, not as a replacement of the teacher guided learning but as a complement to other anatomical learning resources (Trelease, 2002). The aim of this research was to investigate the impact of the use of these resources on student's in-class engagement and experience in the study of gross anatomy in a time-compressed block teaching method.

\section{Method}

This study was approved by the Ethics Committee at Victoria University (HRE-17-192). Informed consent was obtained from all students and participation proceeded on a voluntary basis only. This study uses three different computer programs designed for the study of gross anatomy: An@tomedia Online (Eizenberg et al., 2019), AnatomyTV (Primal-Pictures, 2019) and Thieme (Gilroy et al., 2019), as in-class learning support programs within the newly adopted "Block model" of teaching delivery at Victoria University.

A total of 179 first year students enrolled in the Human Biomedicine /Bioscience degree (HBBM, HBBS) in 2019 participated in the study with 58 respondents. Functional Anatomy of the trunk (unit code RBM1100) is a first year, first semester unit. The curriculum deals with the gross anatomy of the human trunk.

\section{The block teaching model}

Functional Anatomy of the Trunk is taught within the First Year College (FYC) incorporated within the framework of the "block teaching model" which was adopted at the beginning of 2018. The block teaching model is a time-compressed method of intensive teaching in which the entire curriculum of a unit is taught and assessed over 4 weeks, and in which lectures and tutorials are replaced with workshops.

The teaching of the curriculum occurs over eleven 3-hour workshops, consisting of small class sizes of between 20-35 students, in which the composition of the class both in student number and in enrolment stays the same for the duration of the block. Students are strongly recommended to attend the workshops, offered to a maximum of three workshops for each week of the block. The whole block is taught and assessed by 1-3 staff members. Following the theory workshop sessions, students are required to attend a laboratory component consisting of up to nine individual laboratory sessions of 2 hours duration. These laboratory sessions are sequential from the previously scheduled theory workshop session, in which one deals with the theory and the other with practical component of the same topic. Similar to the workshops, a maximum of 3 laboratories are scheduled each week. The laboratory sessions are located in an anatomy laboratory specifically designed for the instruction of gross anatomy and use mainly prosected cadaveric material, articulated skeletons, selected individual bones, anatomical models as well as dissected and/or potted cadaveric specimens (of various appropriate dissections), located on gurneys throughout the laboratory. Typically, there are a maximum of up to 33 students in each laboratory session supported by one demonstrator.

\section{Learning protocol employed during each workshop in which the CAL was used}

The same teaching protocol or lesson plan was applied to each of the 11 three hour theory workshops. At the beginning of each class, the teacher would set the specific student-centred CAL activity task, to be completed from within each of the chosen computer programs during that particular workshop session. This was followed by a teacher-guided introduction, where a specific subject topic from the prescribed curriculum was presented for up to 20-30 minutes 
using diagrams from Gray's Anatomy (Drake, Vogl, \& Mitchell, 2019). Students were then encouraged to engage in a student-centred activity (20-30 minutes) consisting of a hard-copy worksheet consisting of specific matching anatomical images obtained from Thieme (Gilroy et al., 2019), which required them to identify and label or explain relationships of an anatomical structure or function. An example of a worksheet page is shown in Figure 1.

Students were asked to individually visit specific screen pages from An@tomedia Online, AnatomyTV and Thieme, chosen to be the subject mirror-image activities of each other. They were provided with a brief demonstration on their functionality at the beginning of each class and students were encouraged to bring their own electronic devices. Access to the computer programs used occurred via the library portal on the University website, freely available to all enrolled students. They were then asked to slowly work through each work screen (4-8 work screens) from each program and then to compare them with each other during this process (2030 minutes). An example of a screen page from each of the 3 programs used for the study of the anatomy of the heart is shown in Figure 2 (A-F). They were then given time (15-20 minutes) to discuss their individual experiences and impressions with others in their group (randomly allocated 4-6 students). This was aimed at getting them to articulate their impression of the differences to others sitting at their tables.

Students enrolled in this unit were informed of and encouraged to access and use the resources, An@tomedia Online (Eizenberg et al., 2019), AnatomyTV (Primal-Pictures, 2019) and Thieme (Gilroy et al., 2019), in and outside of class time. They were provided with a brief demonstration on their functionality at the beginning of each anatomy unit and students were encouraged to bring their own electronic devices. Access to the computer programs used occurred via the library portal on the University website, freely available to all enrolled students. Apart from the use of the CAL programs students also had available to them, the use of the prescribed textbook, comprehensive study notes for each workshop, a separate hardcopy worksheet for each session, H5P drop and drag learning interactives (Rekhari \& Sinnayah, 2018) appropriate to the topic, short film clips and end of workshop quizzes. 


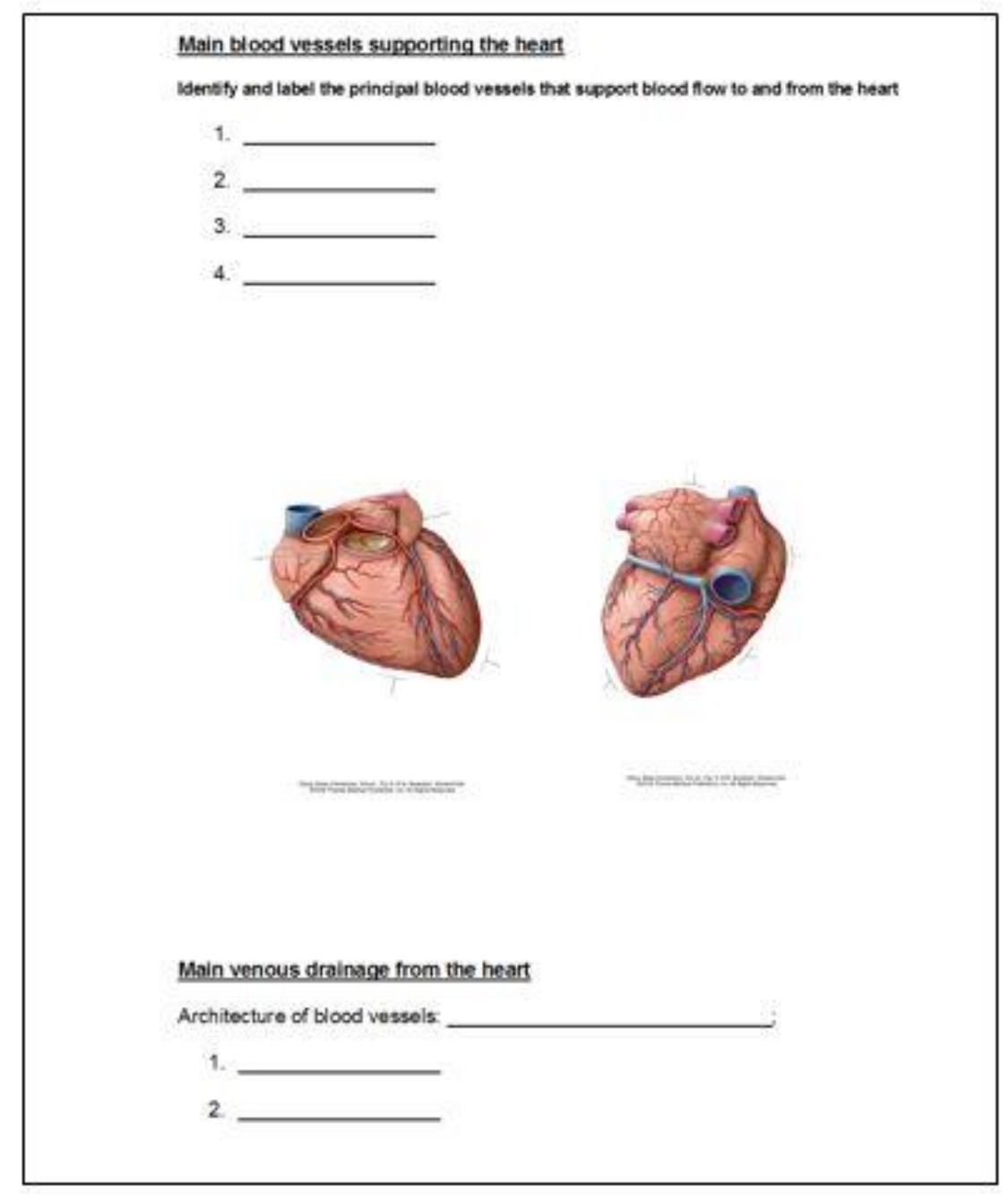

Figure 1. Example of student-centred worksheet from Thieme 


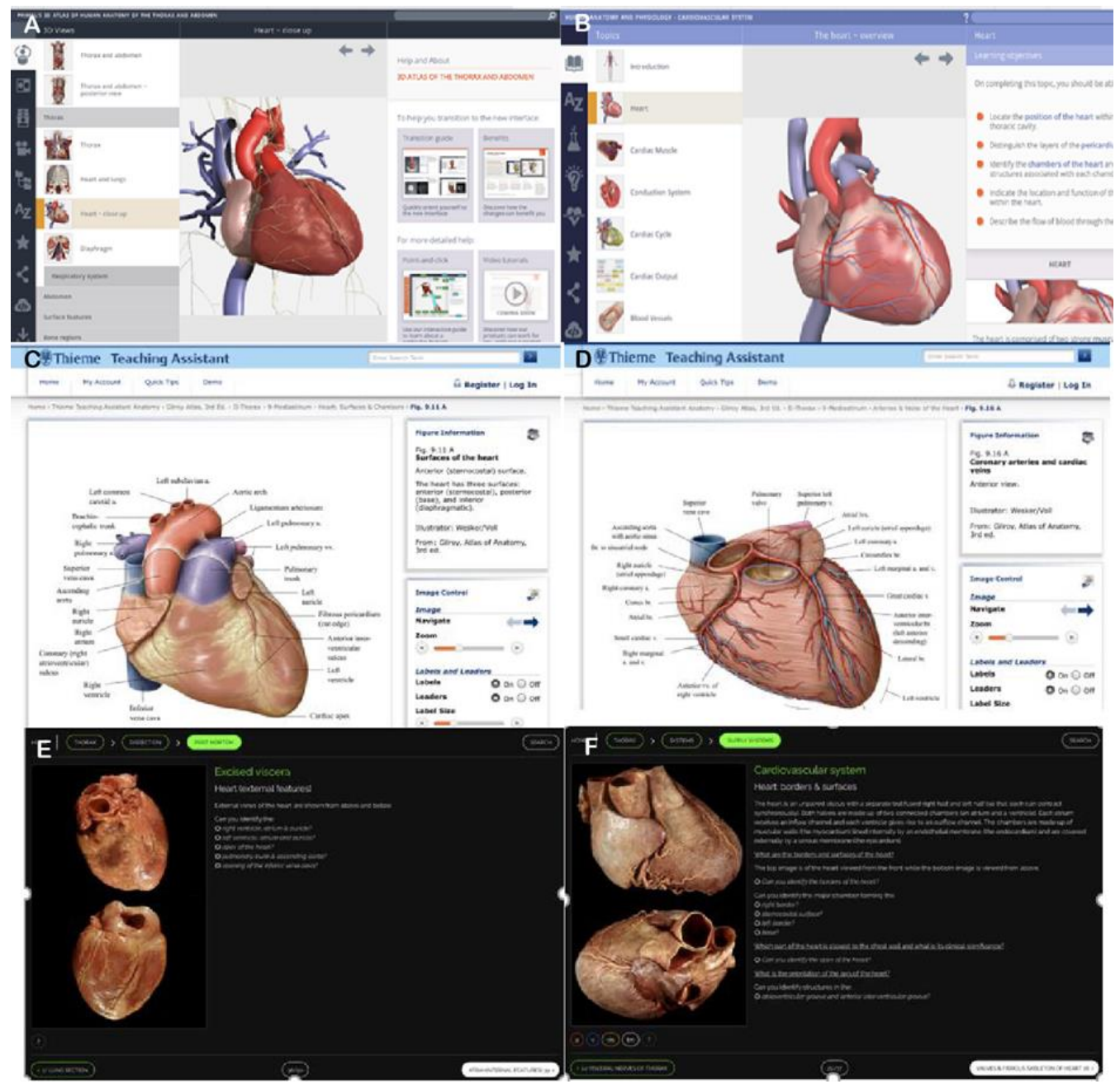

Figure 2: Example screen of 2A) and 2B) AnatomyTV, 2C) and 2D) Thieme and 2E) and 2F)An@tomedia Online computer programs used for the teaching of gross anatomy in RBM1100 Functional Anatomy of the Trunk

\section{Learning protocol used during each gross anatomy laboratory}

Each theory workshop was followed by a $2 \mathrm{hr}$ gross anatomy laboratory session. During a typical laboratory, students (maximum number 33 per laboratory) would begin with an introduction (15-20 minutes) providing demonstrator-guided learning, leaving approximately 100 minutes for exploration of the learning resources on display. Students were encouraged to work together in smaller groups (3-4) studying specific examples appropriate to the topic and cadaveric specimens located on gurneys throughout the laboratory using a laboratory protocol as a guide. During the laboratory sessions, students were encouraged to access and use the CAL resources to assist with their learning. At the completion of each laboratory session, students needed to complete a short knowledge check. This consisted of 10 short-answer response questions in which students were asked to identify a specific structure on a cadaveric specimen, which had been marked with a pin (flag race). The laboratory attendance was compulsory and the laboratory protocol needed to be submitted, marked by the demonstrator and returned to the student as feedback. The laboratory component constituted $40 \%$ of the total grade available.

\section{Survey}

An anonymous opinion-based survey (via Qualtrics) was conducted at the end of each of 3 consecutive anatomy teaching blocks of semester 1 in 2019, and sent to all students enrolled in 
Functional Anatomy of the Trunk. The survey consisted of 17 closed questions and 1 openended question: "Do you have any other comments?" The survey was distributed via e-mail, and or VU collaborate (VU's teaching platform) using the Qualtrics software. All students enrolled in the unit were invited to participate in the project. The opinion-based survey asked students to rank the value of each of the computer-based gross anatomy learning activities in order of 1 to 3 (the top ranking of 1 and the bottom ranking of 3). In addition, a smaller number of students and teaching staff were interviewed. The open-ended item was analyzed and recorded. Written responses from informal interviews from staff or students interviews were manually processed and analyzed.

\section{Results}

\section{Student's perceptions of their own skills and the purpose of studying anatomy}

Results indicate that prior to beginning their study of the anatomy units, most students reported some lack of confidence in relation to their current anatomy knowledge, in response to the question asked: "How confident are you with your Anatomy knowledge before starting this unit? (Figure 3). It can be seen in Figure 3, that very few students reported high levels of confidence $(5.2 \%)$, with most students reporting that they were somewhat confident (37\%), or ambivalent in regards to their own knowledge. Despite the variation in confidence reported, all students in the sample however, did report that they enjoy studying anatomy and that it interests them, with 59.6\% strongly affirming, 38.6\% agreeing and $1.8 \%$ reporting ambivalence. No students within the population reported that they found studying anatomy unenjoyable or uninteresting. Furthermore, $80.7 \%$ of the sample when asked if it was important to study, anatomy responded definitely agree or agree, with once again, $0 \%$ of the student population responding with disagreement to this statement.

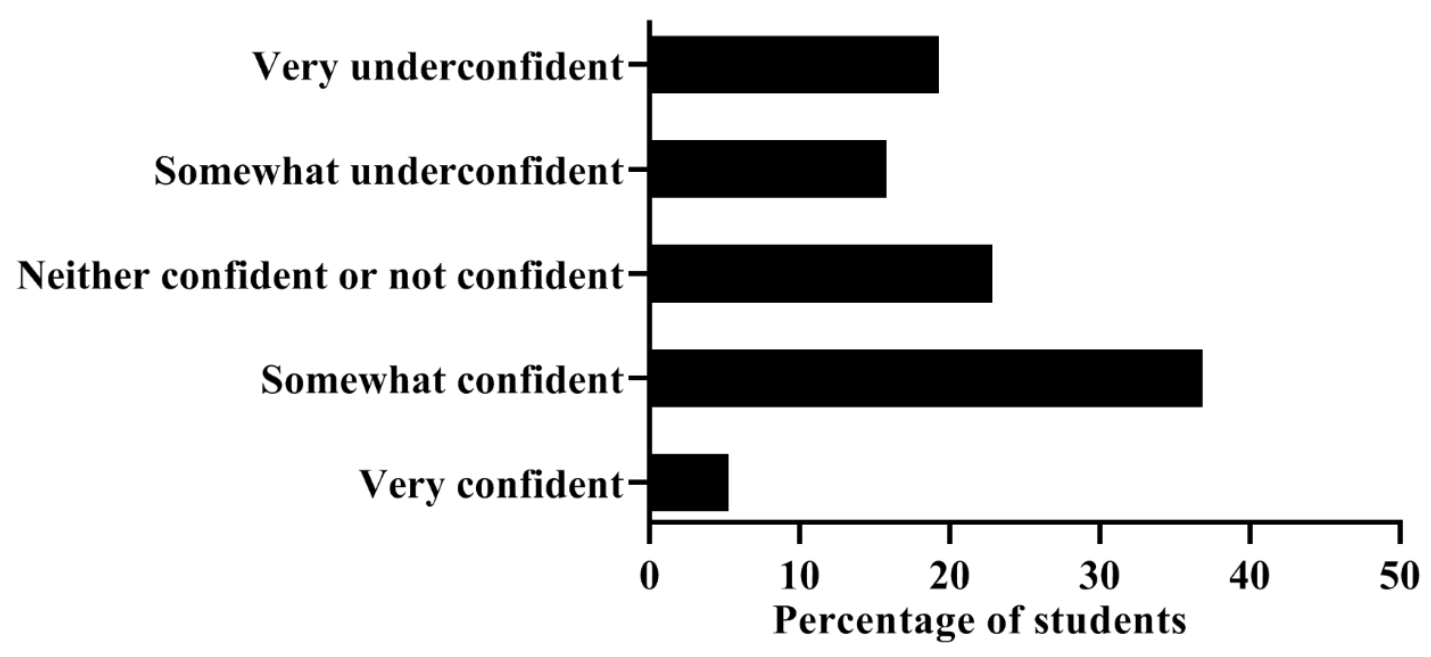

Figure 3: Student's perceptions of their own skills and the purpose of studying anatomy

Most students reported that they found the computer based anatomy programs beneficial in supporting their learning of content, with $94.8 \%$ acknowledging their usefulness. The results of their opinions on each of the programs is depicted below in Table 1. 
Table 1: Student perceptions of the CAL programs

\begin{tabular}{|c|c|c|c|c|c|}
\hline & Best Experience & $\begin{array}{l}\text { Most } \\
\text { Friendly }\end{array}$ & User & Most Utilised & $\begin{array}{l}\text { Inspired In Class } \\
\text { Participation }\end{array}$ \\
\hline Anatomedia & $43.1 \%$ & $48.3 \%$ & & $31.0 \%$ & $39.7 \%$ \\
\hline Anatomy TV & $8.6 \%$ & $15.5 \%$ & & $6.9 \%$ & $17.2 \%$ \\
\hline Thieme & $41.4 \%$ & $29.3 \%$ & & $53.4 \%$ & $37.9 \%$ \\
\hline
\end{tabular}

It is clear from Table 1, that students had a clear preference for both An@tomedia Online and Thieme compared with AnatomyTV for all categories. It can also be noted that user friendliness is not a defining feature in student's choice of programs, as student's reported that An@ tomedia Online was the most user-friendly program. However, they utilised Thieme more frequently. Importantly, all three programs were found to motivate students to engage and participate in class discussions. Furthermore, students were asked to evaluate the content available in each of the three anatomy programs and rank them in order which they thought was the best program for each of the below categories. Student's preferences are shown in Table 2.

Table 2: Student Preferences amongst the CAL programs

\begin{tabular}{llllll}
\hline & $\begin{array}{l}\text { Clarity of } \\
\text { Materials }\end{array}$ & $\begin{array}{l}\text { Ability to } \\
\text { Manipulate } \\
\text { Content }\end{array}$ & $\begin{array}{l}\text { Context of the } \\
\text { Material }\end{array}$ & $\begin{array}{l}\text { Best } \\
\text { Dissection } \\
\text { Diagrams }\end{array}$ & $\begin{array}{l}\text { Incorporation } \\
\text { of } \\
\text { Anatomical } \\
\text { Concepts }\end{array}$ \\
\hline Anatomedia & $30.8 \%$ & $25.0 \%$ & $40.0 \%$ & $62.5 \%$ & $41.7 \%$ \\
Anatomy TV & $23.1 \%$ & $55.6 \%$ & $25.7 \%$ & $8.3 \%$ & $33.3 \%$ \\
Thieme & $46.2 \%$ & $19.4 \%$ & $34.3 \%$ & $29.2 \%$ & $25.0 \%$ \\
\hline
\end{tabular}

Table 2 demonstrates that student's acknowledge that there were strengths and weaknesses to each program, with each program scoring the highest in at least one category. Excluding the ability to manipulate content, An@tomedia Online scored consistently equal to or better than its competitors did. Interestingly, despite being the program students reported using the most, Thieme, performed highest in only one category being clarification of materials.

Table 3 shows written comments from the student feedback open-ended question "Do you have any other comments?" The feedback clearly illustrates that students valued the CAL activity, and included responses such as "Great resource to use An@tomedia" to others directed more specifically at "usability" and the inclusion of "quizzes" into the CAL. 
Table 3: Student comments from the online Qualtrics survey

\begin{tabular}{|c|c|}
\hline Student comments & $\begin{array}{l}\text { - Quizzes are great on TV. Info great on An@ tomedia } \\
\text { Online but hard to search. Diagrams simplest to find } \\
\text { and view on Thieme but lack of other info. Sometimes } \\
\text { confusing to know where to look with all the options. } \\
\text { - It would be better if there's activities that made students } \\
\text { participate in more like quizzes, games that are } \\
\text { anatomy related. } \\
\text { - It would be good if there were active quizzes included } \\
\text { at the end of each session that we could complete in our } \\
\text { own time and showed us what questions we got correct } \\
\text { or wrong. } \\
\text { - Great resource to use anatomedia } \\
\text { The diagrams on the Activity sheets could have been } \\
\text { larger so as to give it more clarity and improved } \\
\text { labelling for better reading and understanding of the } \\
\text { topics involved. } \\
\text { All three anatomy learning support programs were } \\
\text { really helpful. }\end{array}$ \\
\hline
\end{tabular}

Table 4 shows staff feedback to the CAL use, which support the educational benefits of the various programs during class time as student-centred learning activity. There was a preference towards the use of Thieme and progressing to An@tomedia Online, with comments "simple cadaveric images", "easy to navigate" being outstanding features for instructors and students alike. 
Table 4: Staff interview comments on the use of CAL programs

1. From your perspective which of the CAL is the best in promoting student learning during class?

2. Do the CAL fulfill the role of an effective and meaningful in class activity learning tool? Which one is better?

3. Is student learning promoted / enhanced and more effective by the use of CAL? Which one is better?
- I used all 3 programs constantly, Thieme was great to begin a class with; it is clear, simple and correct with little interactivity;

- An@tomedia Online and AnatomyTV were used as in-class activity; An@tomedia Online offered simple cadaveric images;

- Helped with identifying what was ahead in the following lab, in which cadaveric specimens are used all the time;

- An@tomedia Online was designed on a single screen, with simple navigation and essential level interactivity;

- An@tomedia Online promoted further exploration than Thieme and AnatomyTV with questions embedded in the single screen page;

- $\quad$ Thieme was the most widely used resource amongst the students. AnatomyTV least used.

- 'Thieme' is a great way to start an explanation, and good program for in-class

- An@tomedia Online is the next step in moving from a diagram to a real cadaveric image. This builds a realistic expectation of what is ahead in the following wet specimen laboratory.

- AnatomyTV is too small, a little confusing and hard to navigate, in short too complicated.

- 'Thieme' is a fantastic tool that could have worksheets printed from it's contents, and using laminated copies of the sheets, the students could fill out the sheet with whiteboard markers and use these to integrate the knowledge from class and studying wet cadaver specimens further.

- I think that it is a progression from Thieme to An@tomedia Online. Less so to AnatomyTV from what I observed in-class and the comments I got from students.

- ProbablyAn@tomedia Online as it allowed students to see the real cadaveric image and prompted them to investigate further the topic - more student-centred activity

- Student learning is promoted and enhanced while using Thieme and An@tomedia Online.

- Navigation through the pages is user friendly too.

- I believe CAL can supplement learning to provide meaningful and effective integration of content, but the use of wet specimen demonstrations and tasks to be completed in class are fundamental to student education and content understanding. 


\begin{tabular}{|c|c|}
\hline $\begin{array}{l}\text { 4. Do the CAL promote } \\
\text { better student } \\
\text { engagement as an in } \\
\text { class tool? Which one } \\
\text { is better? }\end{array}$ & $\begin{array}{l}\text { - They all increase student engagement (my observation) } \\
\text { - There is clearer more essential information in anatomedia to a topic. } \\
\text { - Students spend a lot of time using Thieme, An@ @omedia Online and AnatomyTV (in this order) making it } \\
\text { an essential tool for doing theory worksheets and understanding the details in gross anatomy. It serves as a } \\
\text { state-of-the-art presentation tool. These online learning resources provide students with flexibility to even } \\
\text { save images and study later. } \\
\text { - Students are most engaged with the online activities when there is a clear relationship between the online } \\
\text { activities and the unit content and assessment tasks. }\end{array}$ \\
\hline $\begin{array}{l}\text { 5. Do you think that the } \\
\text { CAL are a better active } \\
\text { learning tool? Which } \\
\text { one is better? }\end{array}$ & $\begin{array}{l}\text { - They all achieve their aim in active learning. Thieme is a great way to begin and } \\
\text { - Move to An@ tomedia Online for further investigation } \\
\text { - } \quad \text { Anatomy TV is a little too complicated too small work-screen and difficult to navigate } \\
\text { - } \quad \text { As an Educator, it enabled me to utilise these resources for teaching and demonstrating with more clarity. } \\
\text { - Use of the interactive databases supports visual learning and the interactive content can form the basis of a } \\
\text { range of active learning activities. } \\
\text { - The three databases are quite different in terms of their content, interface and navigation. In my view the } \\
\text { strengths of the individual databases are: } \\
\text { - An@ @omedia Online: In my view the strength of this database in terms of a teaching tool is the } \\
\text { comprehensive collection of cadaveric dissection images. This database is also easy to navigate. } \\
\text { AnatomyTV: In my view the strength of this database is the combination of 3D visualization of anatomical } \\
\text { structures with accompanying text. } \\
\text { Additional strengths are the ability to download and link to content. AnatomyTV also has features that enable } \\
\text { integration with learning management systems. } \\
\text { They have the potential to be, but I find no students engage with the CAL unless they are either instructed } \\
\text { to do so, or there is a learning task attached to the utilization of the CAL's. }\end{array}$ \\
\hline
\end{tabular}




\section{Discussion}

The distinctive setting of the educational framework of this study reported here, combining a "teaching block model", with high attendance rates provide a unique and challenging educational paradigm in both space and time (McCluskey et al., 2019). The quality and purpose of student engagement is more important than ever when there is a single teacher to guide the learning, within a single-classroom environment and consisting of small class sizes. Here the quality of the learning materials is one very important contributing factor that will determine the success of how student engage within a framework of active learning (Freeman et al., 2014; Rathner \& Byrne, 2014; Rathner et al., 2013; Sinnayah, Rathner, Loton, Klein, \& Hartley, 2019). From an overall perspective, the computer assisted learning (CAL) program was of particular benefit to students within the new teaching context adopted by Victoria University in 2018 (Bolton, 2018; Matchett, 2018; McCluskey et al., 2019) in providing a meaningful engaging learning medium. Students' positive attitude towards CAL was noted $94 \%$, an observation also supported by previous studies (McNulty et al., 2009).

On a fundamental level, all CAL programs we used were successful in their goal of providing a student-centred learning activity as part of a blended learning environment, as evidenced by the positive student survey feedback and student as well as staff interviews (Table 3 and 4). This is consistent with observations from previous studies (Deslauriers et al., 2011; Freeman et al., 2014; McNulty et al., 2009; Michael, 2006) which show that the development of purposespecific CAL materials which allow self-directed, student-centred learning to be a helpful adjunct to teacher-guided learning . The CAL programs have become increasingly accepted in the study of anatomy (Eizenberg, 2015; Tam et al., 2010), because they offer greater flexibility (24hr access, more cost effective) over cadaver-based anatomy learning (Estai \& Bunt, 2016). It must be emphasised that the use of the CAL programs here was never intended to replace the teacher-guided learning, but rather to act as a complement within the blended learning environment (Khot et al., 2013; Tam et al., 2010). Other benefits associated with the use of CAL include enhancing the quality of the teaching of anatomy by providing alternatives to each anatomical perspective, promoting independent learning, and potentially providing links between theory and practice (Trelease, 2002). In our study, the primary purpose for using CAL was to provide a meaningful engaging activity, which was student orientated and uses up-todate modern technology to enhance the quality of the anatomy learning experience.

In our study, students working in small groups (4-6 randomly allocated) were asked to access the CAL programs on their own computer, and to firstly work individually through specific work screens provided by each program that deal with the same subject topic but differ from each other due to their unique illustrations. Students then compared their experience by discussing this with the experience of others in their small group. This allowed students to process their thinking before engaging in verbalizing their learning through in-group discussion. For each subject topic, the work-screen content from each of the CAL program mirrored the curriculum, as well each other in order to allow such a comparison between the CAL programs to be directly be possible. The learning dynamic had a twofold purpose, firstly a student-centered learning activity provided by each program (examining a topic from three distinctive perspectives); and secondly the subsequent evaluation of this individual learning within the group discussion. Here students through discussion could articulate their learning and formulate the differences between the different CAL programs used.

It is clear that the "best experience", "most user friendly", "most utilised" and "inspired in class participation" was attributed to An@ tomedia Online and Thieme and were clearly the preferred 
"user" programs. User friendliness is an important consideration when choosing a studentcentred learning activity (Eizenberg, 2015). Even though there is a large number of computer assisted learning programs available that aim to support the study of gross anatomy, their comprehensiveness, complexity and user friendliness can limit their applicability (Choi et al., 2008; Estai \& Bunt, 2016).

A similar trend was also observed from the student survey that supported preference for the content of An@tomedia Online and Thieme, compared with AnatomyTV. While for the category "ability to manipulate content", AnatomyTV scored the highest with 55.6\%. Thieme and An@tomedia Online were clearly the preferred program of choice for all other categories including "clarity of materials", "contextualisation of the material", "best dissection diagrams", and "incorporation of anatomical concepts". The reasons for this preference probably relates to the layout of the works screens and the subject content contained within each work screen. As shown in Figure 2 (B-C), work screen from Thieme Figure 2 (D-E), the work screen for An@tomedia, Online are both similar in style, showing the image on the left side, and the menu panels on the right side of the screen. This allows the user to increase the size of the image overall while also containing comprehensive subject content. The image in Figure 2 (A-B) depicting AnatomyTV is centred between two menu panels, one on each side of the program. Additionally, there are two grey bars in which navigation tools are embedded running along the top and bottom of the screen. This formatting leaves a significantly smaller space for the display of the anatomical diagram and impedes the user's ability to comprehend the entire image. Furthermore, the image is less realistic and less clear.

The teaching method should have at its core an ethos to educate students in an engaged manner that enables them to achieve the stated learning outcomes. Students should acquire the skills to be able to interpret and explain reality, in a staged progression: at first being presented with diagrammatic representations in order to become familiar with the structures, and finally being able to identify the structures in cadaveric specimens, applying their knowledge to interpret and explain reality. This learning process should be engaging for the student. Students task in the future is then to apply their anatomical knowledge to a clinical context such as rehabilitation anatomy or in the physiotherapy setting and therefore being able to explain real structures on cadaveric specimens, with the ability to switch between reality and abstract such as an anatomical diagram. From this perspective, An@tomedia Online served the ultimate purpose in providing a realistic image to help process their learning between workshop diagrams and the laboratory, which used cadaveric specimens. An@tomedia Online was the program of choice in rating best for both user experience and the substance of the content together with its relevance. It was simple to navigate, provided simple, clear cadaveric images and supported by interactivity starting with essential facts and then progressing to anatomical concepts, a progression in the level of conceptual difficulty of thinking that students need to acquire when successfully applying their knowledge to a future clinical setting.

We report from student feedback that there was a strong relationship between the relevance of the images or work screens in An@tomedia Online and to the laboratory sessions, which was helpful in assisting them in identifying structures on cadaveric specimens. Because the CAL programs investigated are a comprehensive resource there is no reason that they can't be used with other learning tools, or be easily integrated within other blended learning environments allowing for informal and formal learning experiences to occur simultaneously.

There are a number of limitations to this study, the most significant of which concerns the limited total student pool and number of students who responded to our online survey (32\%). 
While it was evident from personal and other staff observations (Table 4) that most participated in using the programs, this however, did not translate into readiness or willingness to take part in the online survey. While it can be established that at least $32 \%$ of the class used the computer programs (those who completed the survey), we cannot establish whether the students who did not take part in the survey had used the computer assisted learning or not. In addition, as the survey was anonymous, we were unable to determine whether the three programs were used by those students for whom it may have had the greatest benefit, that is, those having the most difficulties with structure identification during laboratory sessions. While feedback from instructors (Table 4) support the benefits associated with the usage of the CAL, student up take remained completely voluntary for both workshop and laboratory sessions.

Further work is needed to consolidate the findings of this study. Although this study was initially aligned to this particular student groups (HBBM and HBBS), there is no reason why the design should not be adaptable for use with other groups (physiotherapy, nursing science) in need of greater or lesser anatomical knowledge. In this vein, these programs could be used or given access to "discipline-specific" anatomy at a higher level than that needed in this study for students mentioned here (e.g., for Undergraduate Medical or osteopathy students) to test their adaptability and flexibility in application. Similarly, it is not possible to provide specific information about which parts of the resources were accessed or how students used the interactive features. Further work will be required to establish how its use might be affected by students' learning approach and attitudes (Svirko \& Mellanby, 2008).

\section{Conclusion}

The conclusions reached in this study based on the student survey results, feedback from student interviews (Tables 1-3) and staff feedback (based on their impressions, Table 4) show that computer-Assisted Learning (CAL) was invaluable in supporting the study of human anatomy within the newly adopted "Block model" of teaching delivery as pioneered at Victoria University. This model has the potential to be similarly effective across a range of educational domains and contexts, particularly if coupled with well selected CAL resources. CAL is an integral component of 'blended learning' where it can be compatible with 'student-centred learning' while complementary with 'teacher-guided learning'.

Collectively, the three chosen CAL programs (An@tomedia Online, AnatomyTV and Thieme) were beneficial in assisting first-year students studying gross anatomy, particularly as these resources encouraged student engagement and enhanced student experience so strongly. It was striking that all students in the sample reported that they enjoyed studying anatomy and that it interested them. However, An@tomedia Online was the most helpful with interpretation of anatomical materials presented. It not only utilised photographic images from cadaver dissection but incorporated anatomical concepts into the learning dynamic. It was also the program students liked most as it provided images of real bodies to help them bridge the learning gap between workshop diagrams and the subsequent laboratory on corresponding cadaveric specimens. 


\section{Acknowledgements}

The authors thank Mr Ethen Kauiers, Dr Rethika Ravi, Ms Suzanne Poliness who assisted with the staff feedback and library data bases information for this study.

\section{Disclosures}

No conflicts of interest, financial or otherwise, are declared by the authors.

\section{References}

Biasutto, S. N., Ignacio C, L., \& Esteban Criado del Río, L. (2006). Teaching anatomy: Cadavers vs. computers? Annals of Anatomy - Anatomischer Anzeiger, 188(2), 187-190. doi:https://doi.org/10.1016/j.aanat.2005.07.007

Bolton, R. (2018). Vice chancellor: More savings needed for university to meet tough new market. Australian Financial Review, 16th September 2018.

Burton, S., \& Nesbit, P. (2002). An analysis of student and faculty attitudes to intensive teaching. Paper presented at the Celebrating teaching at Macquarie North Ryde, NSW: Macquarie University.

Choi, A.-R. A., Tamblyn, R., \& Stringer, M. D. (2008). Electronic resources for surgical anatomy. ANZ Journal of Surgery, 78(12), 1082-1091. doi:10.1111/j.1445-2197.2008.04755.x

Clark, E., \& Clark, P. (2000). The educational show on the road: The promises and pitfalls of intensive teaching in off-shore post graduate coursework programs. International Education, 4(1), 1-13.

Cook, H. (2018). The university shake-up that's lifting students' grades. Sydney Morning Herald, 30th May 2018.

Daniel, E. L. (2000). A review of time-shortened courses across disciplines. College Student Journal, 34(2), 298.

Davies, W. M. (2006). Intensive teaching formats : a review. Issues in Educational Research, 16(1), 1-20.

Deslauriers, L., Schelew, E., \& Wieman, C. (2011). Improved Learning in a Large-Enrollment Physics Class. Science, 332(6031), 862. doi:10.1126/science.1201783

Dixon, L., \& O'Gorman, V. (2019). 'Block teaching' - exploring lecturers' perceptions of intensive modes of delivery in the context of undergraduate education. Journal of Further and Higher Education, 1-13. doi:10.1080/0309877X.2018.1564024

Drake, R. L., Vogl, A. W., \& Mitchell, A. W. (2019). Gray's Anatomy for Students 4th Edition.

Eizenberg, N. (2015). Anatomy and its impact on medicine: Will it continue? The Australasian Medical Journal, 8(12), 373-377. doi:10.4066/AMJ.2015.2550

Eizenberg, N., Briggs, C., Barker, P., \& Grkovic, I. (2019). an @ tomedia. [Database]. . Retrieved from http://www.anatomediaonline.com.

Estai, M., \& Bunt, S. (2016). Best teaching practices in anatomy education: A critical review. Annals of Anatomy - Anatomischer Anzeiger, 208, 151-157. doi:https://doi.org/10.1016/j.aanat.2016.02.010

Freeman, S., Eddy, S. L., McDonough, M., Smith, M. K., Okoroafor, N., Jordt, H., \& Wenderoth, M. P. (2014). Active learning increases student performance in science, engineering, and mathematics. Proceedings of the National Academy of Sciences, 111(23), 8410. doi:10.1073/pnas.1319030111

Gilroy, A. M., McPherson, B. R., Ross, L. M., Schuenke, M., Schulte, E., U., S., \& Baker, E. W. (2019). Thieme Teaching Assistant [Database]. Retrieved from https://www.thieme.com.

Grant David, B. (2001). Using block courses for teaching logistics. International Journal of Physical Distribution \&amp; Logistics Management, 31(7/8), 574-585. doi:10.1108/09600030110402987

Kasworm, C. (2001). "Adult Learner Experiences of an Accelerated Degree Program. Adult Education Research Conference. doi: https://newprairiepress.org/aerc/2001/papers/37

Khot, Z., Quinlan, K., Norman, G., \& Wainman, B. (2013). The relative effectiveness of computer-based and traditional resources for education in anatomy. Anatomical Sciences Education, 6(4), 211-215. doi:10.1002/ase.1355

Kift, S. (2009). Articulating a transition pedagogy to scaffold and to enhance the first year student learning experience in Australian higher education. Final Report for ALTC Senior Fellowship Program. Australian Learning and Teaching Council.

Matchett, S. (2018). Victoria U extends transformative first-year model to all students. Retrieved from Available: [https://campusmorningmail.com.au/news/victoria-u-extends-radical-first-year-model-to-allstudents]. Accessed: 9 th October 2018. 
McCluskey, T., Weldon, J., \& Smallridge, A. (2019). Rebuilding the first year experience, one block at a time. Student Success, 10(1), 1-15. doi:https://doi.org/10.5204/ssj.v10i1.1148

McNulty, J. A., Halama, J., \& Espiritu, B. (2004). Evaluation of computer-aided instruction in the medical gross anatomy curriculum. Clinical Anatomy, 17(1), 73-78. doi:10.1002/ca.10188

McNulty, J. A., Sonntag, B., \& Sinacore, J. M. (2009). Evaluation of computer-aided instruction in a gross anatomy course: A six-year study. Anatomical Sciences Education, 2(1), 2-8. doi:10.1002/ase.66

Michael, J. (2006). Where's the evidence that active learning works? Advances in Physiology Education, 30(4), 159-167. doi:10.1152/advan.00053.2006

Primal-Pictures. (2019). Anatomy.TV. [Database] In Retrieved from https://primalpictures.com.

Rathner, J. A., \& Byrne, G. (2014). The use of team-based, guided inquiry learning to overcome educational disadvantages in learning human physiology: a structural equation model. Advances in Physiology Education, 38(3), 221-228. doi:10.1152/advan.00131.2013

Rathner, J. A., Hughes, D. L., \& Schuijers, J. L. (2013). Redesigning a core first year physiology subject in allied health to achieve better learning outcomes. Int J Innov Sci Math Educ, 21, 40-55.

Reeves, R. E., Aschenbrenner, J. E., Wordinger, R. J., Roque, R. S., \& Sheedlo, H. J. (2004). Improved dissection efficiency in the human gross anatomy laboratory by the integration of computers and modern technology. Clinical Anatomy, 17(4), 337-344. doi:10.1002/ca.10245

Rekhari, S., \& Sinnayah, P. (2018). H5P and Innovation in Anatomy and Physiology Teaching. In D. Wache and D. Houston (Eds.), Research and Development in Higher Education: (Re)Valuing Higher Education, 41, $191-205$

Scott, P. A. (2003). Attributes of high quality intensive courses. . New Directions for Adult and Continuing Education, 97, 29-39.

Sinnayah, P., Rathner, J. A., Loton, D., Klein, R., \& Hartley, P. (2019). A combination of active learning strategies improves student academic outcomes in first-year paramedic bioscience. Advances in Physiology Education, 43(2), 233-240. doi:10.1152/advan.00199.2018

Svirko, E., \& Mellanby, J. (2008). Attitudes to e-learning, learning style and achievement in learning neuroanatomy by medical students. Medical Teacher, 30(9-10), e219-e227. doi:10.1080/01421590802334275

Tam, M. D. B. S., Hart, A. R., Williams, S. M., Holland, R., Heylings, D., \& Leinster, S. (2010). Evaluation of a computer program ('disect') to consolidate anatomy knowledge: A randomised-controlled trial. Medical Teacher, 32(3), e138-e142. doi:10.3109/01421590903144110

Trelease, R. B. (2002). Anatomical informatics: Millennial perspectives on a newer frontier. The Anatomical Record, 269(5), 224-235. doi:10.1002/ar.10177 